Supplement of Hydrol. Earth Syst. Sci. Discuss., 11, 11883-11904, 2014

http://www.hydrol-earth-syst-sci-discuss.net/11/11883/2014/

doi:10.5194/hessd-11-11883-2014-supplement

(C) Author(s) 2014. CC Attribution 3.0 License.

(c) (1)

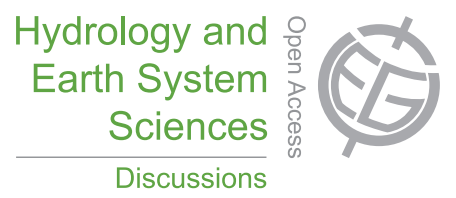

Supplement of

\title{
Technical Note: Surface water velocity observations from a camera: a case study on the Tiber River
}

F. Tauro et al.

Correspondence to: S. Grimaldi (salvatore.grimaldi@unitus.it) 


\section{Water Resources Research}

\section{RESEARCH ARTICLE \\ 10.1002/2014WR015952 \\ Orienting the camera and firing lasers to enhance large scale particle image velocimetry for streamflow monitoring}

Key Points:

- Camera is oriented with its axis perpendicular to the water surface

- Field of view calibration through a system of lasers

- LSPIV accuracy is improved by relating velocity data to tracer density

Correspondence to:

S. Grimaldi,

salvatore.grimaldi@unitus.it

Citation:

Tauro, F., M. Porfiri, and S. Grimaldi (2014), Orienting the camera and firing lasers to enhance large scale particle image velocimetry for streamflow monitoring, Water Resour. Res., 50, doi:10.1002/2014WR015952.

Received 6 JUN 2014 Accepted 3 SEP 2014

Accepted article online 8 SEP 2014

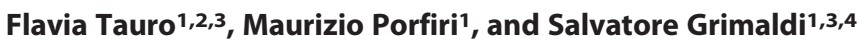 \\ ${ }^{1}$ Department of Mechanical and Aerospace Engineering, New York University Polytechnic School of Engineering, \\ Brooklyn, New York, USA, ${ }^{2}$ Dipartimento di Ingegneria Civile, Edile e Ambientale, Sapienza University of Rome, Rome, \\ Italy, ${ }^{3}$ Honors Center of Italian Universities, Sapienza University of Rome, Rome, Italy, ${ }^{4}$ Dipartimento per I'Innovazione nei \\ Sistemi Biologici, Agroalimentari e Forestali, University of Tuscia, Viterbo, Italy
}

\begin{abstract}
Large scale particle image velocimetry (LSPIV) is a nonintrusive methodology for continuous surface flow monitoring in natural environments. Recent experimental studies demonstrate that LSPIV is a promising technique to estimate flow discharge in riverine systems. Traditionally, LSPIV implementations are based on the use of angled cameras to capture extended fields of view; images are then orthorectified and calibrated through the acquisition of ground reference points. As widely documented in the literature, the identification of ground reference points and image orthorectification are major hurdles in LSPIV. Here we develop an experimental apparatus to address both of these issues. The proposed platform includes a laser system for remote frame calibration and a low-cost camera that is maintained orthogonal with respect to the water surface to minimize image distortions. We study the feasibility of the apparatus on two complex natural riverine environments where the acquisition of ground reference points is prevented and illumination and seeding density conditions are challenging. While our results confirm that velocity estimations can be severely affected by inhomogeneously seeded surface tracers and adverse illumination settings, they demonstrate that LSPIV implementations can benefit from the proposed apparatus. Specifically, the presented system opens novel avenues in the development of stand-alone platforms for remote surface flow monitoring.
\end{abstract}

\section{Introduction}

Recently, considerable interest has been devoted to the development of innovative observational methodologies for improved understanding of hydrological processes [Montanari et al., 2013]. Applications of such novel systems include shallow water flow measurement [Dunkerley, 2003; Lei et al., 2005], stream and rill flow estimation [Berman et al., 2009; Planchon et al., 2005; Stockdale et al., 2008; Tauro et al., 2012a, 2012b], sediment transport and geomorphology monitoring [Hugenholtz and Barchyn, 2011; Mankoff and Russo, 2013], temperature distribution sensing [Selker et al., 2006a, 2006b], areal rainfall estimation [Haberlandt and Sester, 2010], water level measurements [Hut et al., 2010], and noncontact discharge estimation [Costa et al., 2000; Lee et al., 2002; Melcher et al., 2002; Osorio-Cano et al., 2013; Plant et al., 2005; Puleo et al., 2012 ].

Among noncontact river gauging techniques, large scale particle image velocimetry (LSPIV) is a remote surface flow measurement methodology that enables the nonintrusive and continuous characterization of water bodies based on digital image acquisition [Fujita et al., 1997; Bradley et al., 2002]. Such a technique has been utilized to estimate flow discharge in riverine environments [Creutin et al., 2003; Jodeau et al., 2008], flow patterns in limnological ecosystems [Kantoush and Schleiss, 2009], and hydrodynamic features in natural water bodies [Hauet et al., 2009]. Different from river gauging measurements through current meters [Buchanan and Somers, 1969], acoustic Doppler current profilers [Yorke and Oberg, 2002], and dye or chemical tracing [Planchon et al., 2005; Leibundgut et al., 2009], LSPIV does not require either physical sampling or the presence of human operators for data acquisition [Gunawan et al., 2012; Kantoush et al., 2011]. In fact, the methodology allows for safely monitoring extended areas in varying flow regimes, spanning from heavy floods to low flows [Bechle et al., 2012; Tsubaki et al., 2011].

LSPIV is an extension of classical particle image velocimetry (PIV), an optical flow measurement technique that is used in laboratory environments to estimate the instantaneous flow velocity of seeded fluids [Adrian, 1991, 2005; Raffel et al., 2007]. More precisely, LSPIV implementations include: (a) a digital image acquisition system that is often installed at a considerable height (several meters) and angled with respect to the water 
surface to capture extended fields of view (FOVs), (b) highly visible surface flow tracers or patterns, which are carried by the flow, and (c) a processing unit to extract flow velocity from images [Kim et al., 2008; Muste et al., 2008, 2011]. After acquisition, digital images are orthorectified to eliminate distortions introduced by the angled camera and to calibrate image dimensions. Image orthorectification is performed by a photogrammetric relation between fixed ground reference points in the real environment and objects captured in digital images, whereby the accuracy of velocity estimations increases with the number of ground reference points [Hauet et al., 2008]. Specifically, image orthorectification relies on the acquisition of a minimum of four ground reference points, which are typically identified by means of a total station [Hauet et al., 2008]. The identification of such points is deemed as the second major source of inaccuracies after seeding density [Kim, 2006], and represents a considerable hurdle in the implementation of LSPIV in inaccessible environments [Kantoush et al., 2011].

Besides image calibration, ground reference points are utilized to mitigate distortions introduced by the camera tilt angle. In particular, angled cameras introduce perspective distortions, in which near-field objects are better resolved than the far field [Hauet et al., 2008]. Once orthoimages are generated, the motion of surface tracers or flow patterns is used to estimate surface flow velocity through PIV algorithms. In particular, images are subdivided into interrogation windows that are cross-correlated with larger search windows in consecutive frames to estimate the displacements of the tracers. During the process, interrogation windows are translated on a pixel grid superimposed on the pictures, and the flow velocity at each node of the grid is obtained by considering the time delay between consecutive images [Raffel et al., 2007].

LSPIV is a promising flow measurement technique, which allows for pivotal observations in challenging settings, such as surface velocity estimations during extreme floods. LSPIV is potentially a low-cost methodology, whose cost will only reduce in time with technological progress. This should be considered as a major advantage for short-time measurement programs in ungauged settings and environmental monitoring in developing countries. However, standard LSPIV experimental setups present several drawbacks that may hamper practical implementation and increase uncertainty of flow estimation. Specifically, major pitfalls of the technique include the need for ground reference points and the need for floating tracers. These issues result in expensive experimental observations, which hinder continuous measurements in natural settings.

The present manuscript focuses on addressing the need for ground reference points. The dependence of the methodology on the acquisition of ground reference points through GPS or total station dramatically reduces LSPIV applicability. For instance, standard LSPIV may be difficult to implement in case of severe events, where river banks, and thus ground reference points, may be inundated, and in case of inaccessible stream banks in mountainous environments. To extend LSPIV applicability in complex natural settings, this work proposes a mobile and inexpensive sensing system, including a horizontal telescopic mast, a miniature and waterproof GoPro Hero 3 video camera, and a system of two lasers. The laser system creates visible points at a known distance on the water surface, allowing for nonintrusive calibration without dimensioning reference objects in the real environment through ground control points. The setup limits image distortions by mounting the camera at one end of a mast with its axis perpendicular to the water surface. The reduced image coverage due to the camera orthogonality is compensated by the wide-angle GoPro lens, which enables large view capture of the center of the stream. The apparatus can be easily transported and utilized to survey diverse water bodies. Further, to allow for continuous monitoring of large scale environments, the system can be properly customized to be permanently installed underneath bridges or hosted on travelingblock cableways. Future applications of the system may also include its integration in autonomous aerial vehicles for environmental sensing [Hoffer et al., 2014].

To assess the feasibility of the proposed apparatus in surface flow observations, we conduct measurements on two different streams, the Aniene and Tiber Rivers in Rome, Italy. In such study sites, inaccessible river banks, flow conditions, and large scale settings prevent the identification of ground reference points. In addition, the selected experimental settings provide cases for varying flow regimes, illumination conditions, and surface tracer seeding densities. Specifically, the Aniene River exhibits torrential flow regime, abundant ripples induced by water reflections, and a meager number of buoyant tracers. On the other hand, the Tiber River presents lower surface flows, mirror-like reflections, and several inhomogeneously seeded floating objects. While traditional LSPIV is unlikely to be feasible in the selected scenarios, our approach affords the development of usable surface flow velocity maps. To this end, images captured with the novel apparatus 

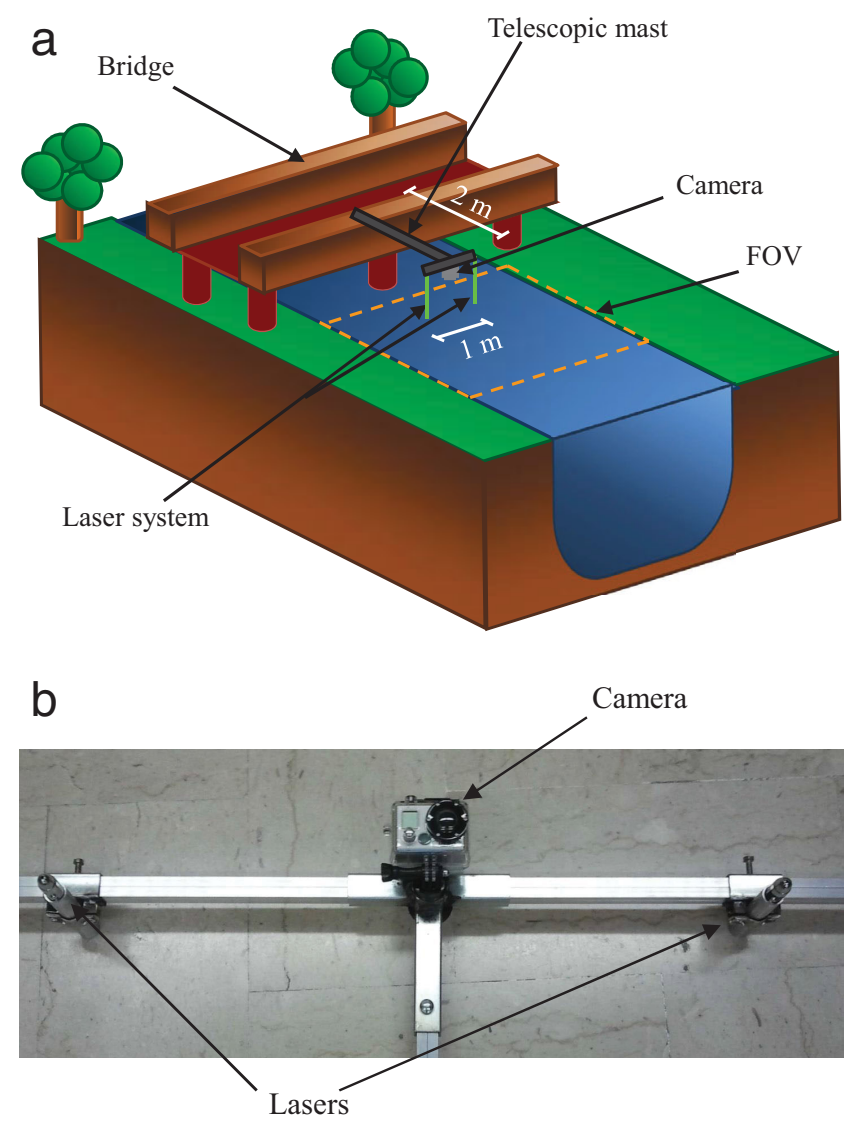

Figure 1. (a) Sketch of the proposed LSPIV implementation and (b) close-up view of the aluminum frame hosting the camera and the laser system. are corrected for camera lens distortion and analyzed with a commercially available PIV software. Surface flow velocity estimations are compared to supervised measurements performed by visually tracking objects floating on the stream surface in acquired images and to rating curves developed by the Ufficio Idrografico e Mareografico (UIM) at Regione Lazio, Italy.

The rest of the paper is organized as follows. Section 2 details the proposed LSPIV experimental apparatus, the study sites, and the imagebased methodologies to estimate surface flow velocity. Section 3 presents LSPIV results for experiments performed in the Aniene and Tiber Rivers; section 4 discusses advantages and criticalities; section 5 is left for conclusions and expected outcomes of the proposed technique.

\section{Materials and Methods}

\subsection{Experimental Apparatus}

The video acquisition system includes a telescopic hollow aluminum bar that can be extended up to $2 \mathrm{~m}$. At one end of the bar, an orthogonal aluminum pole is connected as displayed in Figure 1a. The pole is $1 \mathrm{~m}$ long and hosts two $1 \mathrm{~mW}$ green lasers at its ends, which emit at $532 \mathrm{~nm}$ wavelength. At the intersection of the pole and the bar, a GoPro Hero 3 video camera is encased in a waterproof box, see Figure $1 \mathrm{~b}$. The camera axis is consistently kept perpendicular to the water surface throughout the experiments. Measurements are performed from river parapets by anchoring one end of the bar on the bridge railing and with the pole hanging out parallel to the stream surface.

\subsection{Study Sites}

Two vastly different study sites are selected for LSPIV tests. One class of experiments is conducted on the Aniene River, Italy, where we deal with a torrential flow-regime and free-surface waviness. To assess the feasibility of the methodology in a more challenging scenario, we also execute measurements on the Tiber River, Italy, in low flow conditions and adverse visual settings.

Experiments on the Aniene River are performed at Ponte Salario ( $\left.41^{\circ} 56^{\prime} 22.20^{\prime \prime} \mathrm{N}, 12^{\circ} 30^{\prime} 30.24^{\prime \prime} \mathrm{E}\right)$ at the UIM gauging station APS_17. The station is located on a bridge and is equipped with the ULM20 ultrasonic water meter fabricated by CAE S.p.a. and positioned underneath the midspan of the bridge at the upstream cross section. Water levels are recorded at time intervals of $15 \mathrm{~min}$, and discharges are obtained from the available rating curve. Further, UIM provides the bathymetry for the upstream and downstream cross sections. Due to the larger presence of ripples, foam patterns, and water reflections, the downstream section is selected for LSPIV experiments, and its bathymetry is used for surface velocity estimations from the rating curve. At the time of the video-based measurements, the water meter recorded a level of $1.6 \mathrm{~m}$ and a corresponding discharge of $32.52 \mathrm{~m}^{3} / \mathrm{s}$. 


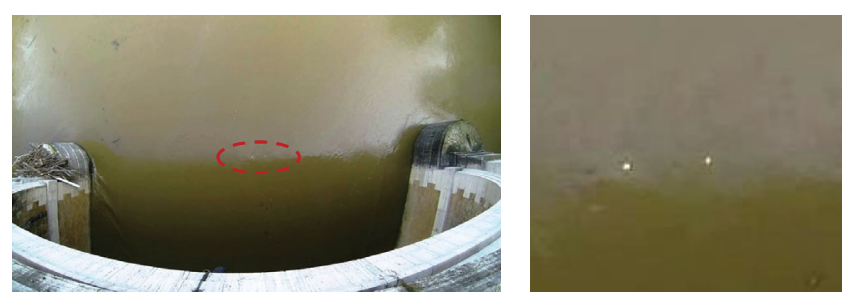

Figure 2. (left) FOV captured in experiments on the Tiber River. The red dashed ellipse encloses the reference points created by the lasers on the water surface. (right) Close-up view of the laser lights.
From graphic estimates on the available bathymetry, the total wetted area for the measured water level is $27.24 \mathrm{~m}^{2}$, and by assuming a velocity correction factor of 0.85 [see Creutin et al., 2003], the average surface velocity is approximately $1.78 \mathrm{~m} / \mathrm{s}$.

Measurements on the Tiber River are conducted at the Ripetta monitoring station ( $46^{\circ} 42^{\prime} 31.30^{\prime \prime} \mathrm{N}, 29^{\circ} 05^{\prime} 34 \mathrm{E}$ ) located at the midspan of the local five-span bridge (Ponte Cavour). The UIM gauging station features a ULM20 ultrasonic water meter located underneath the midspan of the bridge at the upstream cross section, which records level measurements every $15 \mathrm{~min}$. From the available bathymetry for the upstream and downstream cross sections, a discharge of $354.17 \mathrm{~m}^{3} /$ $\mathrm{s}$ is estimated for a level of $7.13 \mathrm{~m}$. By using the available bathymetric data, the total wetted area is estimated to be $680.77 \mathrm{~m}^{2}$, thus leading to an average surface velocity of $0.61 \mathrm{~m} / \mathrm{s}$ for the velocity correction factor set to 0.85 . LSPIV videos are recorded at the center of the midspan of the bridge at both the upstream and downstream cross sections during the falling stage of the flood event that occurred on 14 November 2013, corresponding to meager quantities of floating material transported by the river.

\subsection{Experimental Procedure}

The experimental data set encompasses a set of eight videos recorded at Ponte Salario and 10 recordings acquired at Ripetta. At Ponte Salario, videos of the downstream cross section are acquired at time intervals few minutes apart in the same day. At Ripetta, nine videos are captured from the upstream and one from the downstream cross sections in a time span of $3 \mathrm{~h}$ during the same day. Throughout the experiments, the camera is set to Full HD $(1920 \times 1080$ pixels $)$ resolution and $60 \mathrm{~Hz}$ frame rate. Each video is approximately 2 min long, which corresponds to 7200 images.

Supervised flow velocity is estimated by manually tracking visible floating objects in consecutive sets of images. Specifically, several floaters transiting in the captured fields of view are manually tracked from their entrance in the top of the image to their exit in the bottom of the frame. The time taken by the floaters to transit in the field of view is obtained by computing the number of frames depicting the transit of the objects and from the time lag between consecutive frames. Metric dimensions are assigned to images by equating the pixel distance between laser points from captured frames to the known metric distance $(1 \mathrm{~m})$ of the laser pointers on the aluminum pole, see Figure 2. Specifically, for the Aniene River, the camera FOV captures a region of approximately $60 \times 35 \mathrm{~m}^{2}$, whereas videos performed on the Tiber River monitor an area of $37 \times 21 \mathrm{~m}^{2}$. Flow velocity is estimated to be equal to $4.50-4.70 \mathrm{~m} / \mathrm{s}$ in the Aniene River and to 2 $2.3 \mathrm{~m} / \mathrm{s}$ in the Tiber River as per visual analysis on floaters transiting in the center of both streams. Such values are remarkably different from estimations from the rating curves, which are obtained by dividing the depth averaged streamwise velocity by the velocity correction factor.

\subsection{LSPIV Implementation}

LSPIV is conducted by extracting consecutive sequences of 100 frames from each video. Images are corrected for lens distortion through the GoPro lens intrinsic parameters, as obtained from a preliminary calibration procedure. Further, two alternative methodologies are explored to minimize the effect of the vibrations due to the flexibility of the aluminum bar. Specifically, LSPIV implementations relying on the use of mast-mounted cameras or cableways may be severely affected by wind gusts, which result in slightly varying FOVs and inaccurate flow measurements. Techniques for the removal of unwanted vibrations are similarly developed in Fujita and Hino [2003], Fujita and Kunita [2011], and Pagano [2014] to display identical FOVs in subsequent LSPIV images captured from aerial vehicles and in Wang et al. [2013] to reconstruct images from a dual-camera system. More precisely, one FOV matching methodology entails the cross-correlation between a template image and the sequence of pictures. Images are properly trimmed to depict overlapping FOVs as imposed by LSPIV processing. The template image represents objects on the river banks which are present in every picture. The alternative methodology consists of performing LSPIV on the 


\begin{tabular}{|c|c|c|c|c|c|c|c|c|c|c|}
\hline & \multicolumn{9}{|c|}{ FOV Matching } & \multirow{3}{*}{$\frac{\frac{\text { Velocity Subtraction }}{\text { Full HD }}}{60-32}$} \\
\hline & \multicolumn{5}{|c|}{ Full HD } & \multicolumn{4}{|c|}{ VGA } & \\
\hline & $60-32$ & $30-32$ & $20-32$ & $15-32$ & $60-64$ & $60-32$ & $30-32$ & $20-32$ & $15-32$ & \\
\hline \multicolumn{11}{|c|}{ Aniene } \\
\hline$V_{\mathrm{m}}$ & 2.13 & 2.15 & 1.94 & 1.66 & 1.94 & 1.31 & 1.87 & 1.70 & 1.52 & 1.75 \\
\hline $\begin{array}{l}\beta_{\mathrm{v}} \\
\text { Tiber }\end{array}$ & 0.34 & 0.36 & 0.41 & 0.46 & 0.46 & 0.46 & 0.40 & 0.45 & 0.52 & 0.81 \\
\hline$v_{m}$ & 0.23 & 0.22 & 0.17 & 0.14 & 0.25 & 0.33 & 0.29 & 0.26 & 0.23 & 1.12 \\
\hline$\beta_{\mathrm{v}}$ & 0.87 & 0.86 & 0.82 & 0.71 & 1.08 & 0.82 & 0.90 & 0.96 & 1.04 & 0.95 \\
\hline
\end{tabular}

raw sequence of pictures and then subtracting velocity components relative to fixed objects in the images, such as portions of the bridge, from the velocity of the water surface. PIV analyses are performed with the commercial software EDPIV [Gui, 2013].

\subsection{LSPIV Parameter Selection}

To identify the optimal settings for estimating reliable flow velocities, a preliminary analysis is conducted on one video from each set of experimental acquisitions. Since PIV accuracy is largely influenced by the size of the interrogation window in relation to the homogeneity, size, and motion of the tracers in the field of view, analyses are conducted for varying values of image resolution, size of the interrogation window, and time interval between subsequent images. In addition, both the methodologies described in section 2.4 to mitigate the effect of the mast vibrations are considered. For each experiment, flow velocity values, $v_{\mathrm{m}}$, are obtained from $v_{m}=\frac{1}{N} \sum_{i=1}^{N} \bar{v}_{i}$, where $\bar{v}_{i}$ is the time-average velocity computed at the ith node of the PIV grid and $N$ is the total number of nodes in the grid, see Table 1.

Therein, we also list the index of variation, $\beta_{\mathrm{v}}$, defined as the ratio of the standard deviation of the velocity calculated over the entire FOV to $v_{\mathrm{m}}$, that is, $\beta_{\mathrm{v}}=\frac{1}{v_{\mathrm{m}}}\left[\frac{1}{N} \sum_{i=1}^{N}\left(\bar{v}_{i}-v_{\mathrm{m}}\right)^{2}\right]^{1 / 2}$. This dimensionless parameter indicates the spatial velocity variations in the field of view and should be associated with the heterogeneity of the flow estimations rather than with experimental uncertainties. While the flow is not uniform over the region of interest (lower velocities are expected along the river banks and higher velocities in the central portion of the image), the stream reach does not present riffles, pools, or constrictions and, therefore, the velocity profile is expected to be uniform in cross sections captured in the images. The parameter $\beta_{\mathrm{v}}$ is utilized to provide an estimate of the variability of the velocity values in the field of view, which is highly dependent on the appearance of the water surface and on the occurrence of tracers. Therefore, $\beta_{\mathrm{v}}$ should be regarded as an indicator of the visibility conditions rather than as a parameter to evaluate LSPIV accuracy.

Randomly selected sequences of 100 frames are processed by setting image resolution to Full HD and VGA $(640 \times 480$ pixels). Further, images are subsampled to vary the time interval between pairs of pictures. Full HD sequences of frames are sampled at 0.067, 0.05, 0.033, and $0.0167 \mathrm{~s}$, corresponding to 15 , 20,30 , and $60 \mathrm{~Hz}$ frame acquisition rates. For such image resolution, the interrogation window size is chosen between $32 \times 32$ pixels and $64 \times 64$ pixels, whereas the grid size is always set to half the dimensions of the interrogation window size. VGA sequences of frames are sampled at $0.067,0.05,0.033$, and $0.0167 \mathrm{~s}$, corresponding to $15,20,30$, and $60 \mathrm{~Hz}$ frame acquisition rates, and processed by setting the interrogation window size to $32 \times 32$ pixels. For VGA resolution, larger interrogation window sizes may fail to capture variations in surface patterns and are therefore not considered. Parameters $v_{\mathrm{m}}$ and $\beta_{\mathrm{v}}$ are computed by discarding the false velocity vectors obtained for the river banks and portions of the bridge.

To inform the identification of the most appropriate LSPIV settings, flow estimations from the supervised tracking method (4.50-4.70 m/s for the Aniene River and 2-2.3 m/s for the Tiber River) are considered as 

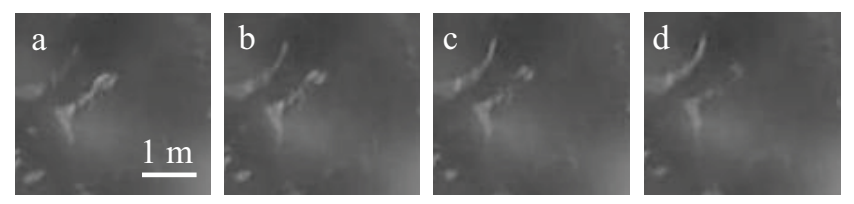

Figure 3. (a-d) Snapshots captured at $60 \mathrm{~Hz}$ of a rapidly evolving surface reflection pattern in the Aniene River. benchmark velocities and, therefore, LSPIV parameters leading to high $v_{\mathrm{m}}$ and low $\beta_{\mathrm{v}}$ in Table 1 are regarded as more reliable. Generally, high values in Table 1 are obtained for high acquisition frame rates and in case of preprocessing

through the FOV matching procedure. Conversely, the velocity subtraction method leads to remarkably low velocities even for image sequences recorded at $60 \mathrm{~Hz}$. At such acquisition rate, consecutive frames precisely depict the evolution of surface patterns, and, therefore, cross-correlation should result in accurate flow estimations. As expected, LSPIV estimates tend to be higher than the values obtained from rating curves $(1.78 \mathrm{~m} / \mathrm{s}$ for the Aniene River and $0.61 \mathrm{~m} / \mathrm{s}$ for the Tiber River), which are affected by numerous uncertainties.

In the case of the Aniene River, higher velocities are found using high frame rate and resolution, whereas flow estimates tend to decrease as the time interval is increased. This behavior is likely due to the deformation of the water reflections in sets of successive images. For instance, Figure 3 depicts snapshots of a surface pattern captured at a time interval of $0.0167 \mathrm{~s}$. From Figures 3a-3d, the light gray shape on the left translates toward the top of the image and rapidly evolves in shape. Such rapid deformations represent serious challenges for PIV analyses since the cross-correlation algorithm may fail to capture pattern similarity in pairs of subsequent images. Specifically, even if the displacement of the tracers is small between successive images in Figure 3, that is, about 2-3 pixels, increasing the time interval in between pairs of images may introduce further errors, since surface patterns may have completely deformed. To alleviate these issues, videos relative to the Aniene River are processed by setting Full HD resolution to fully detect small objects flowing on the surface, $60 \mathrm{~Hz}$ frame rate to account for reflection deformation, and $64 \times 64$ pixels interrogation window size to capture the motion of large scale reflections and foam structures.

On the other hand, videos of the Tiber River are characterized by mirror-like background, slower flows, and instances of sparse floating objects. In this case, acceptable results are obtained by setting a VGA resolution, that preserves the presence of floaters, and increasing the time interval between processed images to $0.033 \mathrm{~s}$, that leads to more accurate PIV estimations in case of slow flow regimes. Both sets of videos are
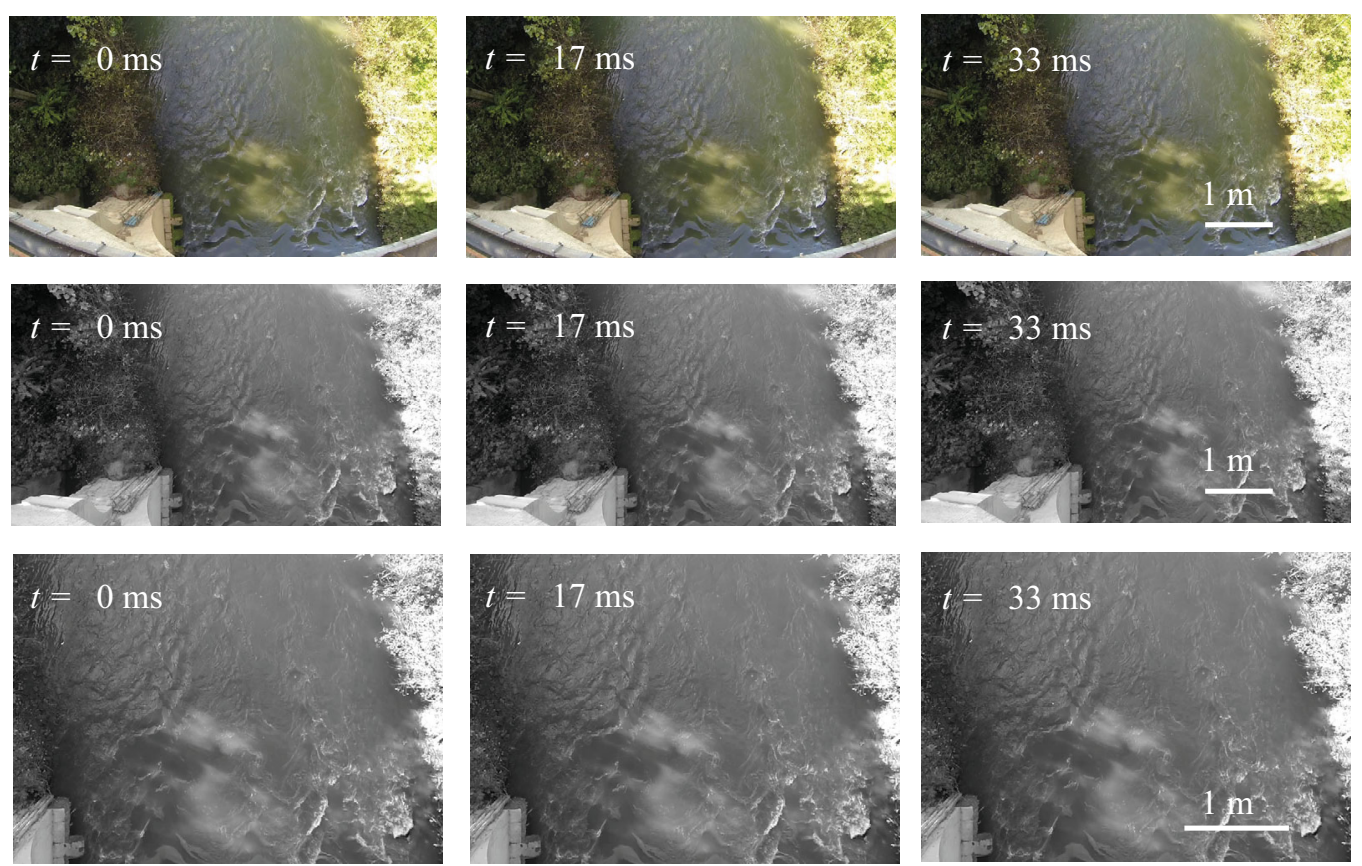

Figure 4. (top) Raw, (middle) undistorted, and (bottom) matched snapshots captured at $60 \mathrm{~Hz}$ of the Aniene River. 


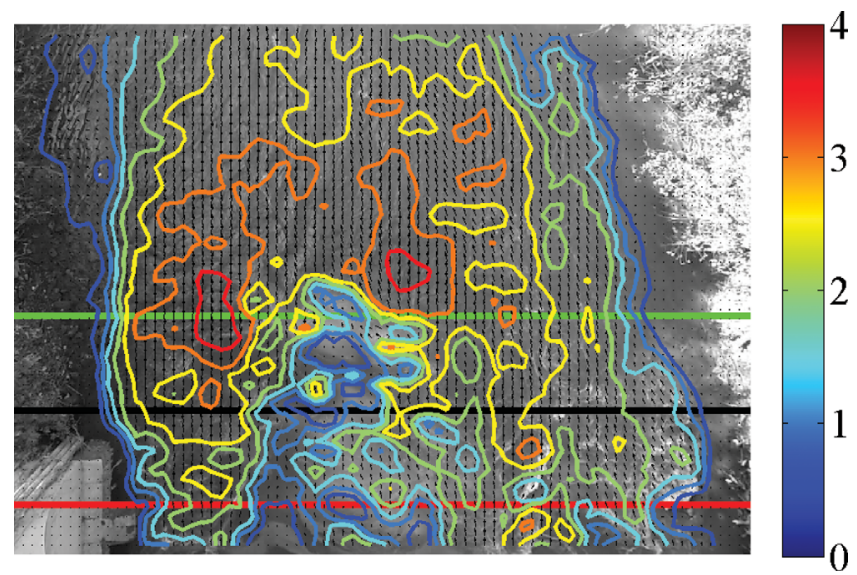

Figure 5. Time-averaged surface flow velocity map of the Aniene River. Values are in $\mathrm{m} / \mathrm{s}$. Red, black, and green lines indicate cross sections at 3, 8, and $13 \mathrm{~m}$ from the bridge, respectively. preliminarily processed through the FOV matching algorithm to further reduce the uncertainty in velocity estimation.

The effect of varying the duration of the observations is also assessed by conducting two PIV analyses on sequences of 300 consecutive frames. Specifically, a sequence of 300 frames of the Aniene River captured at $60 \mathrm{~Hz}$ is analyzed by setting the interrogation window to $64 \times 64$ pixels. By discarding false velocity vectors obtained on the river banks, the parameters $v_{\mathrm{m}}$ and $\beta_{\mathrm{v}}$ are found to be equal to $2.00 \mathrm{~m} / \mathrm{s}$ and 0.44 , respectively. For the representative video of the Tiber River, 300 frames captured at $30 \mathrm{~Hz}$ and VGA resolution are processed. In this case, the parameters $v_{\mathrm{m}}$ and $\beta_{\mathrm{v}}$ are equal to $0.26 \mathrm{~m} / \mathrm{s}$ and 0.88 , respectively. As reported in Table 1, values obtained for longer observations are in line with estimates computed from samples of 100 frames.

\section{Results}

\subsection{Aniene River}

Figure 4 displays a sequence of frames captured at Ponte Salario on the Aniene River recorded at a $60 \mathrm{~Hz}$ frame rate from a representative experiment. In the top row, colored raw images from the GoPro camera are reported, and, in the second row, snapshots are corrected for fish eye lens distortions. Such correction results in gray-scale pictures displaying smaller FOVs. In the last row, images are processed and trimmed to display identical FOVs for PIV analyses. Despite the considerable height of the camera with respect to the water surface (around 15 and $13 \mathrm{~m}$ for the Aniene and Tiber, respectively), the GoPro fish eye lens results in severely distorted FOVs. Further, it is noted that vegetation on the river banks creates large dark areas on the sides of the FOV, while a directly illuminated area is visible in the bottom central region of the pictures. The stream reach presents ripples and waves due to the high flow regime. In the right bottom corner of the images, stationary waves due to the streambed roughness are also identifiable.

Figure 5 depicts a surface flow velocity map for a representative video, where black arrows are velocity vectors and colored contours indicate velocity values in $\mathrm{m} / \mathrm{s}$. Velocity values are computed by averaging PIV estimations in time over the entire sequence of 100 images. No filtering or data postprocessing is

\begin{tabular}{|c|c|c|c|c|c|}
\hline \multicolumn{3}{|c|}{ Aniene } & \multicolumn{3}{|c|}{ Tiber } \\
\hline Test & $v_{\mathrm{m}}$ & $\beta_{\mathrm{v}}$ & Test & $v_{\mathrm{m}}$ & $\beta_{\mathrm{v}}$ \\
\hline 1 & 2.05 & 0.41 & 1 & 0.29 & 0.90 \\
\hline 2 & 2.46 & 0.23 & 2 & 0.31 & 0.90 \\
\hline 3 & 1.94 & 0.46 & 3 & 0.40 & 0.80 \\
\hline 4 & 2.56 & 0.24 & 4 & 0.35 & 0.86 \\
\hline 5 & 2.50 & 0.20 & 5 & 0.30 & 0.73 \\
\hline 6 & 2.57 & 0.24 & 6 & 0.34 & 0.82 \\
\hline 7 & 2.44 & 0.29 & 7 & 0.37 & 0.97 \\
\hline \multirow[t]{3}{*}{8} & 2.17 & 0.37 & 8 & 0.44 & 0.91 \\
\hline & & & 9 & 0.43 & 0.67 \\
\hline & & & 10 & 0.66 & 0.56 \\
\hline
\end{tabular}

${ }^{a}$ Values for $v_{\mathrm{m}}$ are in $\mathrm{m} / \mathrm{s}$, whereas the parameter $\beta_{\mathrm{v}}$ is dimensionless. applied on displayed LSPIV data. Interestingly, PIV analysis accurately captures flow direction whereas low velocity values are obtained along the river banks in proximity of the vegetation. Unrealistic low velocity values are found in the bottom central region of the picture, where sunlight directly illuminates the water surface, see also Figure 4. Unrealistic low flow velocities are also obtained in the bottom right corner of the picture in correspondence of the stationary waves. By averaging the velocity values in the entire spatial domain, a mean flow velocity value of $2.05 \mathrm{~m} / \mathrm{s}$ is obtained with index of variation equal to 0.41 . Similar values are reported in Table 2 for the entire set of experiments.

In Figure 6a, time-averaged surface flow velocity profiles are reported for stream cross sections located at 13, 8, 
a
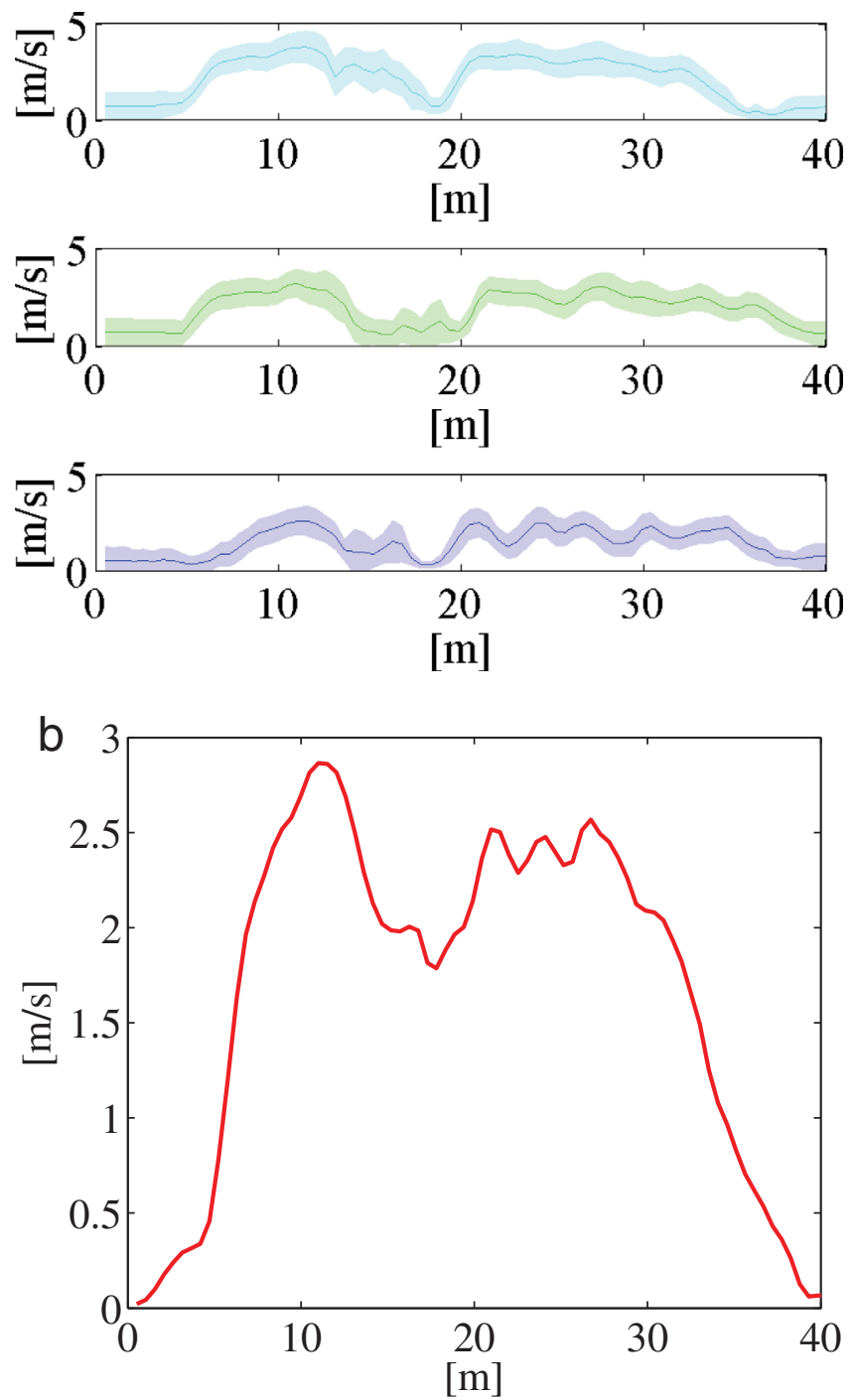

Figure 6. (a) From top to bottom, time-averaged surface flow velocity profiles for the river cross sections at 13,8 , and $3 \mathrm{~m}$ from the bridge obtained from a representative experiment. The origin of the horizontal coordinate in the plots corresponds to the left side river bank in Figure 5. Shaded areas indicate the standard deviation over the sequence of 100 frames analyzed in this experiment. (b) Surface flow velocity profile obtained by averaging with respect to space and time over the entire set of river cross sections captured in the FOV for the same representative experiment. For this experiment, $v_{\mathrm{m}}=2.05 \mathrm{~m} / \mathrm{s}$ and $\beta_{\mathrm{v}}=0.41$. images are homogeneously illuminated and water surface reflections are scarce. Several floating objects and debris are concentrated in the center of the stream. Yet, the limited dimensions of the objects and poor contrast with respect to the homogeneous background reduce the tracer visibility.

Figure 8 presents a time-averaged surface flow velocity map of the Tiber River as obtained from PIV analyses, where each value of the grid is computed by averaging over the selected sequence of frames. Notably, velocity vectors do not describe realistic flow patterns and velocity values are almost everywhere close to zero due to the absence of appropriate tracers. Areas of higher flow velocities are concentrated in the center of the stream where the transit of floating objects is observed. Consistently, the time-averaged velocity value over the entire spatial domain is estimated to be $0.35 \mathrm{~m} / \mathrm{s}$ with index of variation equal to 0.86 . Similar results for the entire set of experiments are illustrated in Table 2. 

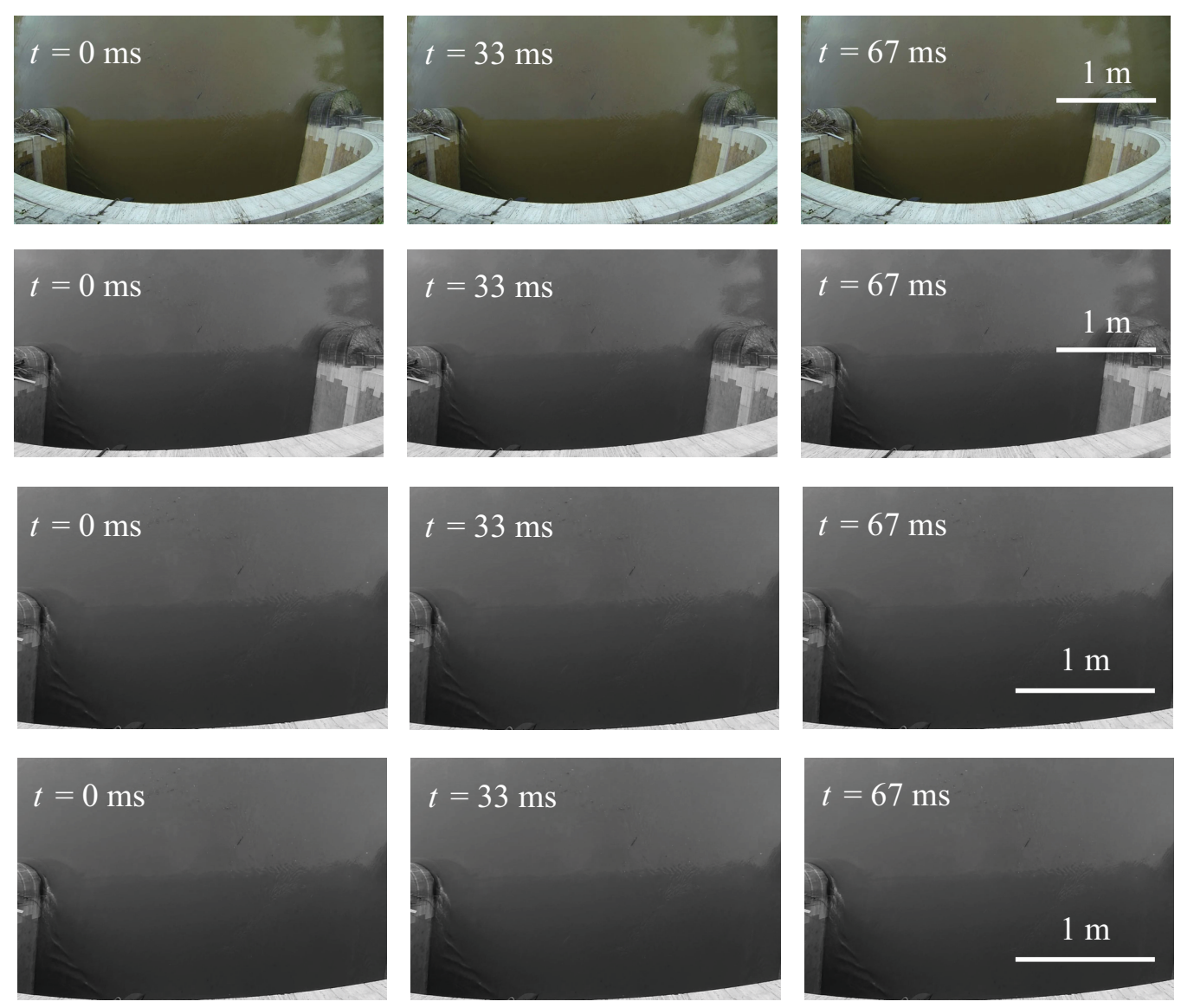

Figure 7. From top to bottom, raw, undistorted, matched, and lower-resolution snapshots of the Tiber River captured at $30 \mathrm{~Hz}$.

Figure 9a reports time-averaged surface flow velocity profiles for cross sections located at 13, 10, and $3 \mathrm{~m}$ from the bridge, where each value on the PIV grid along the river cross section is averaged over the selected sequence of frames. Profiles are obtained for the same representative video analyzed in Figure 8. In this case, velocity values are almost constant for the entire stream span and do not sensibly decrease at the bridge piers. In Figure 9b, the time-averaged surface velocity profile further averaged over the stream cross sections is equal to $1 \mathrm{~m} / \mathrm{s}$ everywhere along the entire cross section.

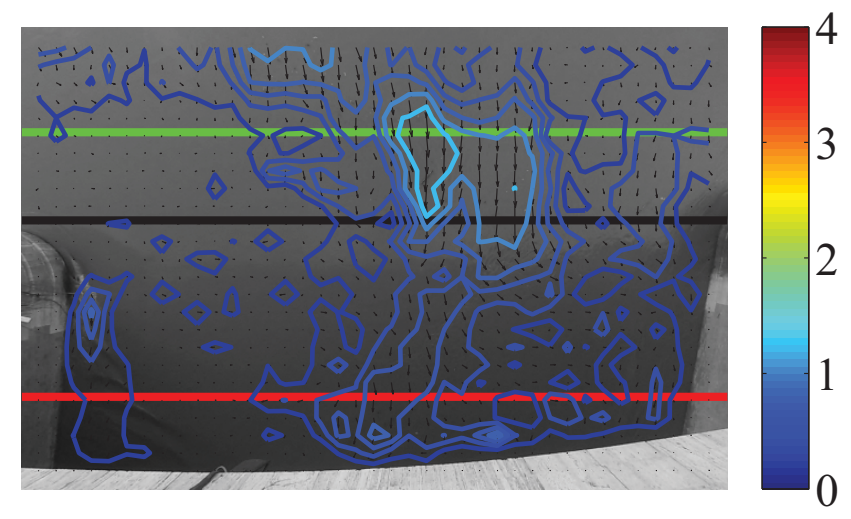

Figure 8. Time-averaged surface flow velocity map of the Tiber River. Values are in $\mathrm{m} / \mathrm{s}$. Red, black, and green lines indicate cross sections at 3,10 , and $13 \mathrm{~m}$ from the bridge, respectively.

\section{Discussion}

While our results can be considered encouraging given the challenging experimental conditions, they draw the attention to two major issues. Specifically, flow velocities are consistently lower than estimations from the supervised methodology, and indices of variation are remarkably high. Flow underestimations are mainly attributed to the inhomogeneous density, or absence, of surface tracers. 
a
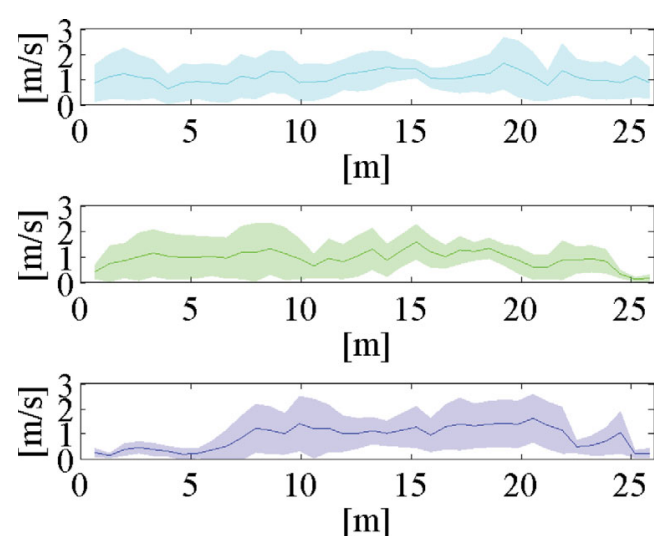

b

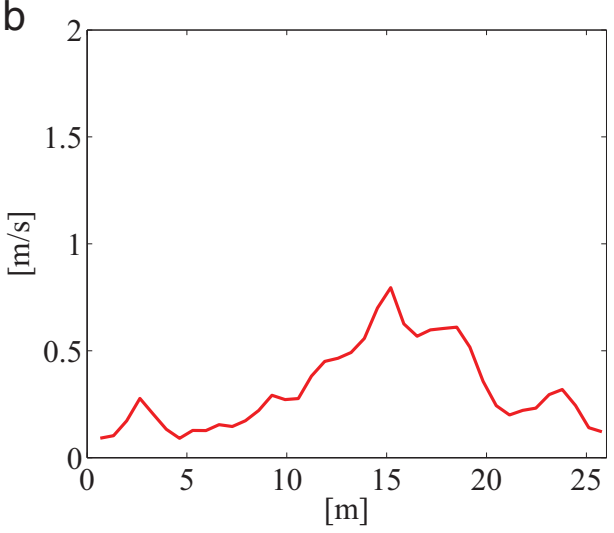

Figure 9. (a) From top to bottom, time-averaged surface flow velocity profiles for the river cross sections at 13, 10, and $3 \mathrm{~m}$ from the bridge obtained from a representative experiment. The origin of the horizontal coordinate in the plots corresponds to the left side river bank in Figure 8. Shaded areas indicate the standard deviation over the sequence of 100 frames analyzed in this experiment. (b) Surface flow velocity profile obtained by averaging with respect to space and time over the entire set of river cross sections captured in the FOV for the same representative experiment. For this experiment, $v_{\mathrm{m}}=0.99 \mathrm{~m} / \mathrm{s}$ and $\beta_{\mathrm{v}}=0.80$.

Indeed, in both experimental data sets, flow velocities that are close to predictions from the supervised analysis are observed at isolated locations in the center of the streams, where floaters are more likely to transit. High flow spatial heterogeneity is attributed to the large areas of null flow values recovered in proximity of the stream sides, in poorly illuminated regions, and in homogeneous visibility zones. For instance, with regards to the Aniene River, even if foam patterns and ripples were observable, several structures were stationary, thus resulting in unrealistic low velocities. In experiments on the Tiber River, given the poor contrast with the background and practical challenges in homogeneously seeding the entire river cross section, little improvements were observed with the deployment of wax particles. Such unrealistic null velocity values highly contribute to lowering spatial averages and to increasing indices of variation in Table 2. Notably, the high variation in flow estimations cannot be attributed to frame sampling time. In case of the Tiber River, the poorly seeded surface leads to images depicting almost identical FOVs and, therefore, sampling frame rate is decreased to $30 \mathrm{~Hz}$ to limit unrealistic null velocities in large portions of the frames. On the other hand, in case of the rapidly deforming floaters in the Aniene River, sampling frame rate is kept to 60 $\mathrm{Hz}$ to prevent the images to be too dissimilar for cross-correlation.

Despite few locations in the stream center, velocity values obtained by averaging PIV data over the entire duration of the experiments also led to significant velocity underestimations. For instance, in Figure 10, we expand on Figure 6 a by reporting boxplots for velocity values obtained at each node of the PIV grid along the selected cross sections for the entire 100 frame sequence. Specifically, edges of the filled boxes indicate the $25 \%$ and $75 \%$ percentiles, circles refer to mean velocity values, error bars display minimum and maximum data points, and squares show outliers. The shaded red horizontal stripes indicate flow velocities estimated through manual tracking on floaters transiting in the center of the stream. From Figures 10a-10c, the distance from the bridge, and therefore from the adversely illuminated area, decreases, thus resulting in generally lower velocity profiles. As expected, the variability and the number of outliers are high, suggesting that velocity estimations depend on the irregular transit of surface tracers and flow patterns. Conversely, in the poorly illuminated area (see values from 16 to $21 \mathrm{~m}$ from the left river bank), the density of outliers decreases, thus indicating a region of critical visibility. Resting upon evidence in Figure 10, we plot in Figure 11 velocity contour and color maps obtained by considering the $85 \%$ percentile rather than averaging velocity values with respect to time. In this case, surface velocities are much closer to estimations from the supervised tracking procedure, while still accurately describing flow regime in the entire FOV. Low flow values faithfully relate to the side of the stream and to the critical visibility area.

While our findings confirm the promise of LSPIV for monitoring water bodies, they indicate that its accuracy is highly dependent on the transit of visible flow patterns. In case of torrential flows, LSPIV is expected to lead to more reliable surface velocity fields than low flows. Nonetheless, the detrimental effect of irregularly transiting tracers and stationary patterns should be accounted for by analyzing variations of the velocity 

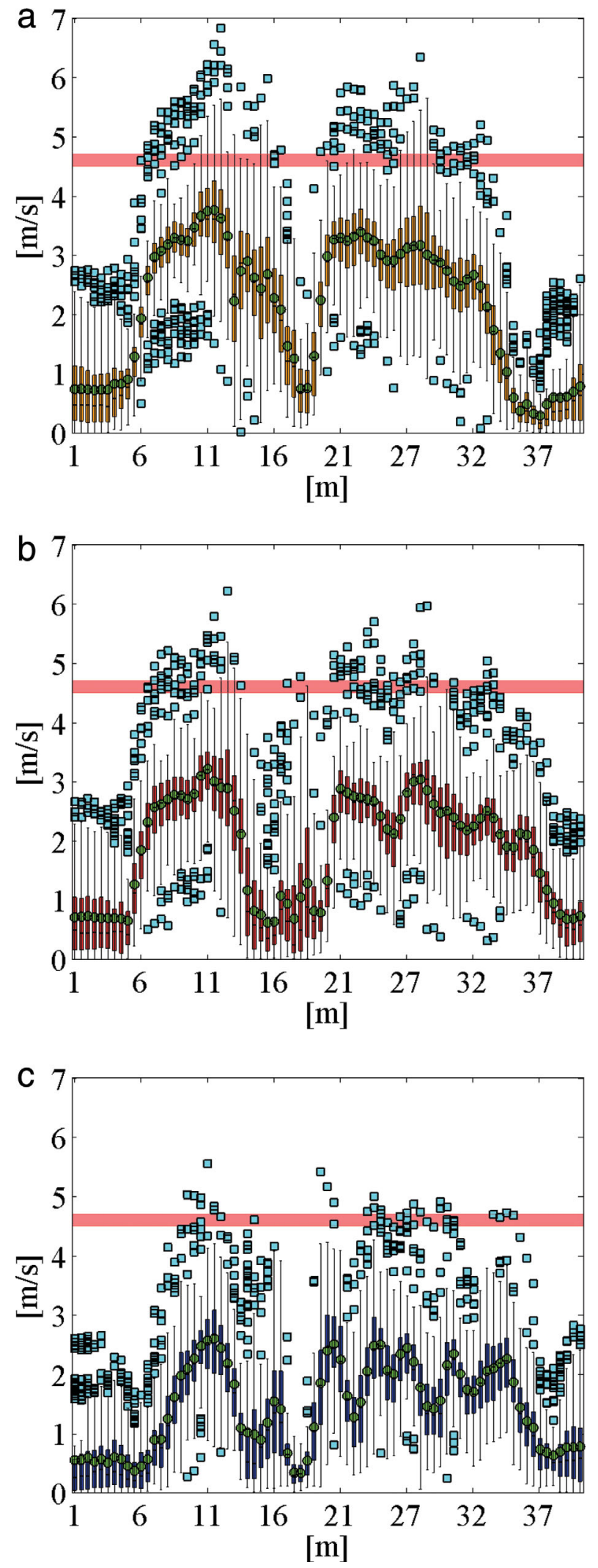

Figure 10. Boxplots for velocity values obtained from PIV analyses at cross sections located at (a) 13, (b) 8 , and (c) $3 \mathrm{~m}$ from the bridge on the Aniene River. Red stripes show supervised benchmark flow values. field estimated by PIV during the time of observation. For this purpose, we expect that sensitivity analyses on the dependence of LSPIV data on flow conditions will aid in the selection of adequate velocity percentiles to systematically create velocity maps analogous to those in Figure 11. These preliminary calibration analyses can also contribute to the improvement of LSPIV-based rating curves [Dramais et al., 2011]. Specifically, LSPIV-based discharge estimations are currently performed on selected cross sections by considering the time-averaged surface velocity values computed at the nodes of the PIV grid. Based on our findings, more accurate estimations may be attained by: (i) studying time variations of velocity data and investigating their relationship with flow conditions, and, therefore, with varying surface seeding densities, (ii) removing null or unrealistic velocities attributable to the absence of tracers or poor illumination in the entire FOV, and (iii) developing a representative cross section by averaging over the stream transects captured in the FOV. Such procedures may be of great advantage in engineering practice for the development of and for comparison to locally available rating curves.

While such measures could improve estimations on the Aniene River, in the low flow conditions experienced on the Tiber River, flow measurements were particularly challenging due to the almost complete absence of clearly visible surface patterns. According to Muste et al. [2008], the accuracy of LSPIV estimations is highly related to the presence 

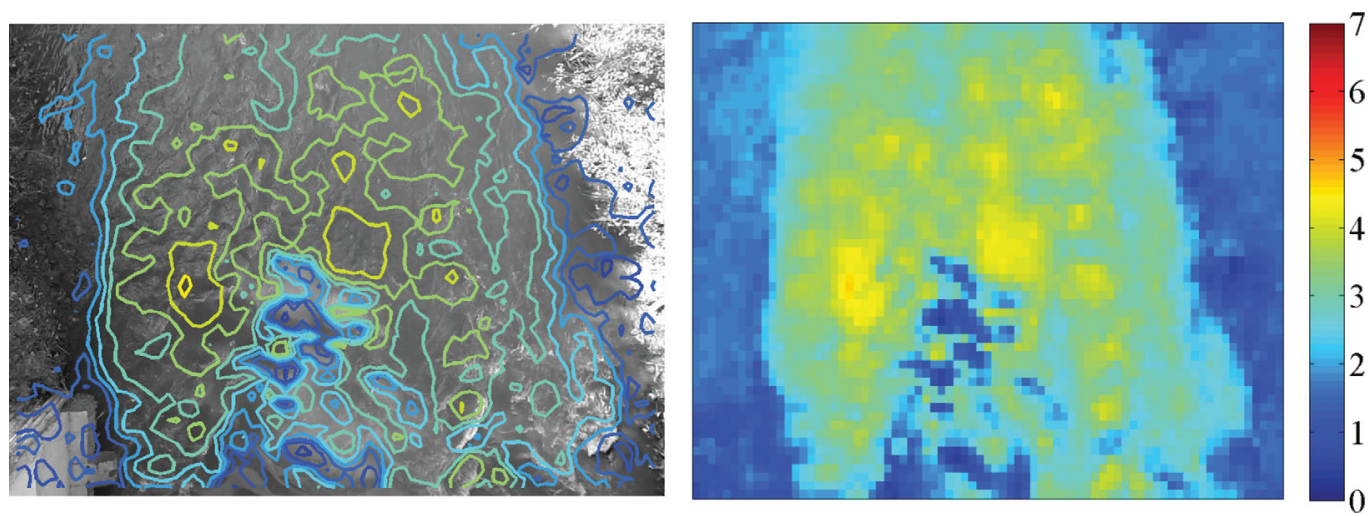

Figure 11. (left) contour and (right) color maps for the $85 \%$ percentile velocity values estimated from PIV. Values are in $\mathrm{m} / \mathrm{s}$.

of properly seeded water surfaces and the combination of tracer-poor visibility and scarce illumination has led to a maximum error of 35\% in flow velocity estimations in Muste et al. [2008] and Kim [2006]. In some instances, artificial seeding has been necessary to compensate for the absence of moving patterns [see for example, Dramais et al., 2011; Meselhe et al., 2004]. Alternatively, LSPIV implementations have been performed in proximity of water falls or bridge piers, thus exploiting surface foam and ripples as tracers [Creutin et al., 2003; LeCoz et al., 2010]. In our study, even manually seeded floaters on the Tiber River were not sufficient to improve velocity estimations, and we expect such challenges to be exacerbated in case of larger FOVs, where massive seeding apparatuses would be necessary. We thus suggest that LSPIV measurements should be preceded by feasibility studies on the identification of proper highly visible tracers to accurately seed the flow. In future studies, we will investigate the combined use of buoyant fluorescent particles [Tauro et al., 2013a, 2012c, 2013b], UV or LED illumination, camera filters, and polarizers to remarkably enhance LSPIV measurements.

\section{Conclusions}

In this paper, we investigated the feasibility of using a novel LSPIV sensing apparatus in challenging natural settings that present varying flow regimes, illumination conditions, and tracer seeding densities. Our inhouse developed system allows for remote image calibration, thus considerably simplifying experimental analyses and image processing. By utilizing such a system, we sought to obtain reasonably accurate flow estimations and aimed at identifying the optimal operational settings of the apparatus to perform measurements in natural environments.

The introduced fish eye camera allowed for observations of surface flow velocity maps of large scale water bodies, and the laser system enabled remote calibration in areas inaccessible to operators and bulky equipment. By providing two reference points on the water surface, laser calibration also reduced eventual inaccuracies associated with ground reference points that do not lie on the same plane of the region of interest. Further, the computationally inexpensive image analysis procedures allowed for rapid estimation of surface flows over large areas. While slight sources of inaccuracies can be attributed to the lens correction and FOV matching procedures, the relative contribution of such uncertainties is compensated by the remarkable distance of the acquisition system from the water surface and through care in handling the telescopic mast. Our results suggest that LSPIV accuracy could benefit from independent analysis on the relationship between time variations of the velocity field and flow conditions, including presence of tracers and flow patterns.

Our research paves the way to completely remote LSPIV implementations, where the presence of human operators for the acquisition of ground control points is prevented. Ongoing work is being devoted to the validation of the apparatus against standard LSPIV in controlled environments, where stream banks are easily accessible for GPS and total station surveying, and in case of smaller scale settings, where alternative techniques such as intrusive probes can be deployed in the current. In addition, the design and development of an unmanned aerial platform for LSPIV measurements is currently being addressed, where an orthogonal camera compensates for image geometric undistortion and a laser system allows for remote 
calibration [Pagano, 2014]. This work thus provides a preliminary feasibility analysis of the potential of using self-sufficient aerial sensing platforms for large scale and continuous surface flow velocity measurements in inaccessible areas.

\section{Acknowledgments}

This work was supported by the American Geophysical Union Horton Research grant for PhD students, by the MAE project 2014 Italy-USA PGR00175, by the Honors Center of Italian Universities, and by the National Science Foundation under grant BCS1124795. The authors thank Andrea Petroselli, Giorgio Olivieri, Roberto Rapiti, and Giuliano Cipollari for help with the experiments and Francesco Mele, Domenico Spina, and Luigi D'Aquino from UIM for providing ULM20 water level measurements and rating curves. The authors are also greatly thankful to Rolf Hut, three anonymous Reviewers, and the Associate Editor for precious comments that have helped improving the work and its presentation.

\section{References}

Adrian, R. J. (1991), Particle-imaging techniques for experimental fluid-mechanics, Annu. Rev. Fluid Mech., 23, 261-304.

Adrian, R. J. (2005), Twenty years of particle image velocimetry, Exp. Fluids, 39(2), 159-169.

Bechle, A., C. Wu, W. Liu, and N. Kimura (2012), Development and application of an automated river-estuary discharge imaging system, J. Hydraul. Eng., 138(4), 327-339.

Berman, E. S. F., M. Gupta, C. Gabrielli, T. Garland, and J. J. McDonnell (2009), High-frequency field-deployable isotope analyzer for hydrological applications, Water Resour. Res., 45, W10201, doi:10.1029/2009WR008265.

Bradley, A. A., A. Kruger, E. A. Meselhe, and M. V. I. Muste (2002), Flow measurement in streams using video imagery, Water Resour. Res., 38(12), 1315, doi:10.1029/2002WR001317.

Buchanan, T. J., and W. P. Somers (1969), Discharge measurements at gaging stations, U.S. Geol. Surv. Tech. Water Resour. Invest., Book 3 , Chap. A8, $65 \mathrm{pp}$.

Costa, J., K. Spicer, R. Cheng, F. Haeni, N. Melcher, E. Thurman, W. Plant, and W. Keller (2000), Measuring stream discharge by non-contact methods-A proof-of-concept experiment, Geophys. Res. Lett., 27(4), 553-556.

Creutin, J. D., M. Muste, A. A. Bradley, S. C. Kim, and A. Kruger (2003), River gauging using PIV techniques: A proof of concept experiment on the lowa River, J. Hydrol., 277(3-4), 182-194.

Dramais, G., J. LeCoz, B. Camenen, and A. Hauet (2011), Advantages of a mobile LSPIV method for measuring flood discharges and improving stage-discharge curves, J. Hydroenviron. Res., 5(4), 301-312.

Dunkerley, L. D. (2003), An optical tachometer for short-path measurement of flow speeds in shallow overland flows: Improved alternative to dye timing, Earth Surf. Processes Landforms, 28(7), 777-786.

Fujita, I., and T. Hino (2003), Unseeded and seeded PIV measurements of river flows video from a helicopter, J. Visualization, 6(3), $245-252$.

Fujita, I., and Y. Kunita (2011), Application of aerial LSPIV to the 2002 flood of the Yodo River using a helicopter mounted high density video camera, J. Hydroenviron. Res., 5(4), 323-331.

Fujita, I., M. Muste, and A. Kruger (1997), Large-scale particle image velocimetry for flow analysis in hydraulic engineering applications, J. Hydraul. Res., 36(3), 397-414.

Gui, L. (2013), EDPIV—Evaluation software for digital particle image velocimetry, The University of lowa, lowa City, la. [Available at http:// Icgui.net.]

Gunawan, B., X. Sun, M. Sterling, K. Shiono, R. Tsubaki, P. Rameshwaran, D. Knight, J. Chandler, X. Tang, and I. Fujita (2012), The application of LS-PIV to a small irregular river for inbank and overbank flows, Flow Meas. Instrum., 24, 1-12.

Haberlandt, U., and M. Sester (2010), Areal rainfall estimation using moving cars as rain gauges a modelling study, Hydrol. Earth Syst. Sci., $14,1139-1151$

Hauet, A., J. D. Creutin, and P. Belleudy (2008), Sensitivity study of large-scale particle image velocimetry measurement of river discharge using numerical simulation, J. Hydrol., 349(1-2), 178-190.

Hauet, A., M. Muste, and H.-C. Ho (2009), Digital mapping of riverine waterway hydrodynamic and geomorphic features, Earth Surf. Processes Landforms, 34(2), 242-252.

Hoffer, N. V., C. Coopmans, A. M. Jensen, and Y. Chen (2014), A survey and categorization of small low-cost unmanned aerial vehicle system identification, J. Intelligent Robotic Syst., 74(1-2), 129-145.

Hugenholtz, C. H., and T. E. Barchyn (2011), Laboratory and field performance of a laser particle counter for measuring aeolian sand transport, J. Geophys. Res., 116, F01010, doi:10.1029/2010JF001822.

Hut, R. W., S. V. Weijs, and W. M. J. Luxemburg (2010), Using the Wiimote as a sensor in water research, Water Resour. Res., 46, W12601, doi: 10.1029/2010WR009350.

Jodeau, M., A. Hauet, A. Paquier, J. Le Coz, and G. Dramais (2008), Application and evaluation of LS-PIV technique for the monitoring of river surface velocities in high flow conditions, Flow Meas. Instrum., 19(2), 117-127.

Kantoush, S. A., and A. J. Schleiss (2009), Channel formation during flushing of large shallow reservoirs with different geometries, Environ. Technol., 30(8), 855-863.

Kantoush, S. A., A. J. Schleiss, T. Sumi, and M. Murasaki (2011), LSPIV implementation for environmental flow in various laboratory and field cases, J. Hydroenviron. Res., 5(4), 263-276.

Kim, Y. (2006), Uncertainty analysis for non-intrusive measurement of river discharge using image velocimetry, PhD thesis, Grad. Coll. of the Univ. of lowa, The University of lowa, lowa City, la.

Kim, Y., M. Muste, A. Hauet, W. F. Krajewski, A. Kruger, and A. Bradley (2008), Stream discharge using mobile large-scale particle image velocimetry: A proof of concept, Water Resour. Res., 44, W09502, doi:10.1029/2006WR005441.

LeCoz, J., A. Hauet, G. Pierrefeu, G. Dramais, and B. Camenen (2010), Performance of image-based velocimetry LSPIV applied to flash-flood discharge measurements in mediterranean rivers, J. Hydrol., 394(1-2), 42-52.

Lee, M.-C., C.-J. Lai, J.-M. Leu, W. J. Plant, W. C. Keller, and K. Hayes (2002), Non-contact flood discharge measurements using an X-band pulse radar (I) theory, Flow Meas. Instrum., 13(5-6), 265-270.

Lei, T., X. Weisheng, Z. Jun, L. Zhen, and Z. Qingwen (2005), Method for measuring velocity of shallow water flow for soil erosion with an electrolyte tracer, J. Hydrol., 301(1-4), 139-145.

Leibundgut, C., P. Maloszewski, and C. Külls (2009), Tracers in Hydrology, Wiley-Blackwell, Oxford, U. K.

Mankoff, K. D., and T. A. Russo (2013), The Kinect: A low-cost, high-resolution, short-range 3D camera, Earth Surf. Processes Landforms, 38(9), 926-936.

Melcher, N. B., et al. (2002), River discharge measurements by using helicopter-mounted radar, Geophys. Res. Lett., 29(22), 2084, doi: $10.1029 / 2002$ GL015525.

Meselhe, E. A., T. Peeva, and M. V. I. Muste (2004), Large scale particle image velocimetry for low velocity and shallow water flows, J. Hydrol. Eng., 130(9), 937-940. 
Montanari, A., et al. (2013), Panta Rhei everything flows: Change in hydrology and society-The IAHS scientific decade 2013-2022, Hydrol. Sci. J., 58(6), 1256-1275.

Muste, M., I. Fujita, and A. Hauet (2008), Large-scale particle image velocimetry for measurements in riverine environments, Water Resour. Res., 44, W00D19, doi:10.1029/2008WR006950.

Muste, M., H.-C. Ho, and D. Kim (2011), Considerations on direct stream flow measurements using video imagery: Outlook and research needs, J. Hydroenviron. Res., 5(4), 289-300.

Osorio-Cano, J. D., A. F. Osorio, and R. Medina (2013), A method for extracting surface flow velocities and discharge volumes from video images in laboratory, Flow Meas. Instrum., 33, 188-196.

Pagano, C. (2014), Development of a quadricopter for environmental flow observations, MS thesis, N. Y. Univ. Polytech. Sch. of Eng., Brooklyn, N. Y.

Planchon, O., N. Silvera, R. Gimenez, D. Favis-Mortlock, J. Wainwright, Y. Le Bissonnais, and G. Govers (2005), An automated salt-tracing gauge for flow-velocity measurement, Earth Surf. Processes Landforms, 30(7), 833-844.

Plant, W. J., W. C. Keller, and K. Hayes (2005), Measurement of river surface currents with coherent microwave systems, IEEE Trans. Geosci. Remote Sens., 43(6), 1242-1257.

Puleo, J. A., T. E. McKenna, K. T. Holland, and J. Calantoni (2012), Quantifying riverine surface currents from time sequences of thermal infrared imagery, Water Resour. Res., 48, W01527, doi:10.1029/2011WR010770.

Raffel, M., C. E. Willert, S. T. Wereley, and J. Kompenhans (2007), Particle Image Velocimetry. A Practical Guide, Springer, N. Y

Selker, J. S., et al. (2006a), Distributed fiber-optic temperature sensing for hydrologic systems, Water Resour. Res., 42, W12202, doi:10.1029/ 2006WR005326.

Selker, J. S., N. vandeGiesen, M. Westhoff, W. Luxemburg, and M. B. Parlange (2006b), Fiber optics opens window on stream dynamics, Geophys. Res. Lett., 33, L24401, doi:10.1029/2006GL027979.

Stockdale, R. J., S. J. McLelland, R. Middleton, and T. J. Coulthard (2008), Measuring river velocities using GPS river flow tracers (GRiFTers), Earth Surf. Processes Landforms, 33(8), 1315-1322.

Tauro, F., S. Grimaldi, A. Petroselli, and M. Porfiri (2012a), Fluorescent particle tracers for surface flow measurements: A proof of concept in a natural stream, Water Resour. Res., 48, W06528, doi:10.1029/2011WR011610.

Tauro, F., S. Grimaldi, A. Petroselli, M. C. Rulli, and M. Porfiri (2012b), Fluorescent particle tracers in surface hydrology: A proof of concept in a semi-natural hillslope, Hydrol. Earth Syst. Sci., 16(8), 2973-2983.

Tauro, F., G. Mocio, E. Rapiti, S. Grimaldi, and M. Porfiri (2012c), Assessment of fluorescent particles for surface flow analysis, Sensors, 12(1111), 15827-15840.

Tauro, F., M. Porfiri, and S. Grimaldi (2013a), Fluorescent eco-particles for surface flow physics analysis, AIP Adv., 3(3), 032108

Tauro, F., E. Rapiti, J. F. Al-Sharab, L. Ubertini, P. Grimaldi, and M. Porfiri (2013b), Characterization of eco-friendly fluorescent nanoparticle doped-tracers for environmental sensing, J. Nanopart. Res., 15(9), 1884.

Tsubaki, R., I. Fujita, and S. Tsutsumi (2011), Measurement of the flood discharge of a small-sized river using an existing digital video recording system, J. Hydroenviron. Res., 5(4), 313-321.

Wang, F., B. Xu, M. Xu, J. Shi, L. Jia, and C. Li (2013), A large-scale particle image velocimetry system based on dual-camera field of view stitching, Sensors Transducers, 157(10), 234-239.

Yorke, T. H., and K. A. Oberg (2002), Measuring river velocity and discharge with acoustic Doppler profilers, Flow Meas. Instrum., 13(5-6), 191-195. 